\title{
Assessing workplace chemical exposures: the role of exposure monitoring $\dagger$
}

\author{
Martin Harper \\ Exposure Assessment Branch, Health Effects Laboratory Division, National Institute for \\ Occupational Safety and Health, 1095 Willowdale Rd. MS-3030, Morgantown, WV, \\ 26505, USA.
}

Received 13th November 2003, Accepted 17th February 2004

First published as an Advance Article on the web 11th March 2004

Occupational exposure is the condition of being subjected through employment to a chemical, physical, or biological agent, or to a specific process, practice, behavior, or organization of work. Exposure to a chemical agent is typically the contact of that agent with the outer boundary of a subject, such as the respiratory system, skin, or digestive system. In occupational hygiene we are most concerned with exposure through the respiratory system, although, increasingly we are concerned with the results of dermal exposures, including those exposures to the skin that can be transferred to the mouth and digestive system. This presentation will detail methods available for assessing personal exposures to chemicals through monitoring. The results from monitoring can then be compared to established guidelines and regulations, although this is not the only rationale for making measurements. These monitoring methods are currently used around the world to establish the benchmark hazard from which risk to the worker can be predicted. The presentation will describe the general techniques for assessing exposures to the respiratory system from chemical gases and vapors, chemical dusts, and exposures to the skin from bulk chemicals or chemical contamination of surfaces. For respiratory exposures, direct-reading instruments are available for spot measurements, and for monitoring short-term fluctuations in concentration. However, most standards and regulations are based on time-integrated (time-weighted average) exposures, requiring longer-term integrative methods. Therefore, the specific focus of this review will be the methods available for full work-shift sampling. For gases and vapors this will include taking whole-air samples in canisters or polymer bags, or concentration of chemicals by absorption in liquids or adsorption on solid sorbents, with subsequent chemical analysis. Chemical concentration can take place by pumping air through the sorbing media, or by allowing molecules to diffuse to the sorbent surface. Transfer of the collected chemicals to the analytical instrumentation can be accomplished using solvent displacement and injection, or through the application of heat to bring the collected molecules back into the vapor phase. For particles, the particle size is important as this determines the site of deposition in the lungs, and so time-integrated sampling on filters using various types of size-selective samplers is preferred. Finally, some techniques that have been used to assess the potential for chemical contamination of the skin are presented. Biomonitoring is another tool that can be used to assess exposure, and the results are more relevant to dosimetric considerations than exposure. Biomonitoring is a complex subject worthy of a separate review, and will be considered only briefly here.

Dr Harper's education spans geology, analytical chemistry, pollution control, and environmental science. After gaining his PhD from the London School of Hygiene and Tropical Medicine for research into air sampling methods development he moved to the USA and worked for a leading manufacturer of air sampling instruments for eleven years. He is a Certified Industrial Hygienist, and has taught at Universities in Alabama, West Virginia, and Jamaica. His current interests include sampling and analysis of coarse dusts including wood and metals,

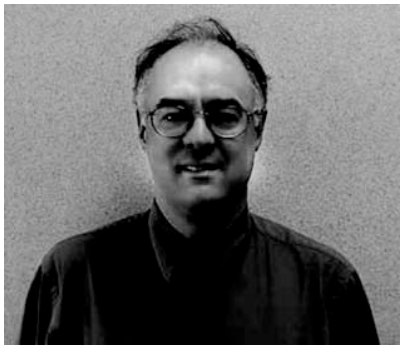

Martin Harper metalworking fluids, asbestos and silica, as well as indoor air chemistry, exhaled breath monitoring and exposure assessment strategies. He has served on many technical committees concerning air monitoring, and currently is Secretary of the ASTM D22.04 and Chair of the ISO TC146/SC2 subcommittees on workplace air quality.

$\dagger$ Presented at the Second International Conference on Environmental, Health and Safety Aspects Related to the Production of Aluminium (EHSARPA), St. Petersburg, Russia, 26 September-1 October, 2003.

\section{Introduction}

When considering the discipline of exposure assessment it is first necessary to define what is meant by exposure. Exposure is part of the process whereby occupational disease is caused and manifested, forming the link between generation of a potential hazard within the environment and the presentation of that hazard to the body. Occupational exposure is the condition of being subjected through employment to a chemical, physical, or biological agent, or to a specific process, practice, behavior, or organization of work.

A narrower definition, which is particularly appropriate to chemical hazards, is that exposure can be considered as the contact of a hazard with the outer boundary of a subject, including the respiratory system, the skin, and the digestive system. Assessment of exposure to chemicals may be necessary for several purposes. One common application is in tracing linkage from disease to possible causative agents by screening for the presence of specific chemicals ("hazard identification"), which may also include assessment of the hazard dosage to the target organ. Exposure assessments are used to investigate complaints of symptoms in workplaces, and, for this purpose, the investigator may make measurements of symptomatic and control populations. Another use for exposure assessment includes determining the need for, and effectiveness of 
engineering controls, in which case measurements are made before and after implementation or changes in the control technology. Perhaps the most common motivation for assessments is the need to support allegations of non-compliance with regulations and standards covering exposure limits ("exposure evaluation"). Typically, measurements are targeted using professional judgment to those considered to have the highest exposures. A database of measurements resulting from any of the above needs does not lend itself to the evaluation of large workplaces or an entire industry for epidemiological purposes, and neither can it be used to recreate accurate historical exposures for these populations. Instead a system is required for randomly assessing exposures amongst the population over a long time period ("exposure estimation").

This tutorial review will cover "exposure characterization", which, for chemical agents, is the determination of concentrations to which a worker is exposed, either instantaneously, or over some shorter (15-minutes) or longer (8-hour) period, usually as a time-weighted average. The exact period selected will depend on the type of injury, for example, acute irritation versus chronic systemic poisoning, and also the pharmacokinetics of detoxification. In making measurements for any of the purposes listed above, study design is critical - the "who, where, when and how often" of monitoring, and different purposes require a different strategy. In addition, these purposes may require different degrees of accuracy in the measurement. This has been fairly well established for specific tasks, such as compliance monitoring, but more research is needed in this area.

Exposure measurements are best when they can be related to the dose. Since workplace contamination can vary significantly both in time and space, close attention must be paid to monitoring the route of exposure as close to the worker as possible. For airborne contamination measurements, these should be made as close as possible to the breathing zone (commonly assumed as within $30 \mathrm{~cm}$ of the center line between nose and mouth). Nevertheless, dramatic differences between exposures measured at different points over the head and upper torso may be observed if the atmosphere is at all heterogeneous, as is almost always the case for particulate exposures, and is frequently the case for vapor exposures from point sources. Again, more research is needed in this area. Government agencies have put enormous effort into developing and validating measurement methods. Although these methods generally are required to have an accuracy or uncertainty level applicable to regulation or litigation, the agencies have been very mindful of issues including cost, and worker acceptability. In the last few years, many of these methods have made their way to the internet, where they are available for free, including those from US government agencies, such as the National Institute for Occupational Safety and Health (NIOSH), the Occupational Health and Safety Administration (OSHA), and the Environmental Protection Agency (EPA):

NIOSH: http://www.cdc.gov/niosh/nmam.html

OSHA: http://www.osha.gov/dts/sltc/methods

EPA (ambient): http://www.epa.gov/ttn/amtic

EPA (emissions): http://www.epa.gov/ttn/emc

and the French INRS: http://www.inrs.fr/metropol.

Some other methods resources include the Methods for the Determination of Hazardous Substances series from the UK Health and Safety Executive, the German Analytische Methoden and BIA Arbeitsmappe Messung von Gefahrstoffen, or the Swedish Principer och Methoden.

There is an unfortunate paradigm that has developed in recent years that making measurements is expensive. A rational analysis of the cost breakdown and cost-benefit is needed. Validation of the techniques often is covered by government agencies (how many other products receive this benefit?). The capital equipment required for sampling (pumps, directreading instruments) is large, but typically similar to the costs of other office equipment, and often with a much longer life-span. Even with maintenance and calibration costs included, amortization over a large number of samples makes the cost per sample small. The sampling media (filters, sorbent tubes) typically are low-cost and disposable. The laboratory analysis probably is the largest contributor to the overall cost, yet laboratory automation continues to bring these costs down. For comparison, consider the cost per sample is frequently less than a single drug prescription for a sick employee, or less than the cost of one day's absence from work for that employee. Where costs may be considered to become excessive is when the employment of an "expert" is factored into the equation. The cost/benefit issue of having a hygiene professional on site is certainly arguable. Industry frequently spends more on consultants in other business areas, and perhaps for less benefit, while the value of professional observations of processes and work practices from the perspective of health and safety often is underestimated. Nevertheless, the making of measurements can be delegated to lower-paid technicians, or trained employees. Also, it may be possible to develop cheaper, less accurate methodologies for characterizing overall workplace conditions where between-worker variation is much larger than the uncertainty of the method.

The final choice of method involves many factors, including whether the method is stipulated by regulation, the measurement period is short or long-term, the concentrations are high or low, the sample is meant to be personal or site-specific, the availability, ease of use, or cost of equipment in the field and in the laboratory, and the required accuracy of the result. It is often the case that more than one method could be considered to give a "valid" measurement. Measurements are made to support decisions. These decisions may have health consequences and costs associated with them. Therefore, a degree of confidence that the result is sufficiently accurate ("fit-forpurpose") is highly desirable. Government validation of the technique, manufacturer's maintenance of a quality manufacturing system, and demonstration of competence by analytical laboratories through quality system accreditation and proficiency testing are important factors in ensuring quality measurements.

The following methods for assessing exposure to gases and vapors will be described:

Direct-reading instruments

Detector tubes

Whole air samplers

Bubblers (liquid sorbents)

- Solid sorbent samplers

$\checkmark$ Active and passive sampling

Solvent and thermal desorption

$\checkmark$ Chemical reaction

And also the following methods for assessing exposures to aerosols:
Direct-reading instruments
- Impingers
Filters and cassettes
- Size-selective samplers
$\checkmark$ Respirable
Thoracic
$\checkmark$ Inhalable

In addition, there are brief discussions of methods for sampling mixed phases, and of methods for assessing exposures via the skin and ingestion, and biological methods of exposure assessment ("biomonitoring"). Finally, there is a note concerning method validation and accuracy. For more comprehensive coverage of any of the topics dealt with here, the reader is advised to consult the wider literature, and some guidance to this is available in the bibliography at the end. The reader further should note that the present author is most familiar with the techniques in common usage in the USA, and reference to specific samplers or sampling methodologies may 
reflect this bias. However, it is the case that both the US NIOSH and OSHA have contributed tremendously to the range of available techniques, and have been generous in making these available to practitioners throughout the world, and many have been adopted internationally.

\section{Methods for assessing exposures to gases and vapors}

\section{Direct reading instruments}

The need for instant analysis of the atmosphere is particularly important when dealing with situations immediately dangerous to life and health, including situations of potentially flammable atmospheres, oxygen deprivation, or acutely toxic concentrations, all of which may occur in conjunction with areas of restricted air exchange, often referred to as confined spaces. Instruments that make these measurements do not need to be very sensitive, but do need to be very accurate and robust, as the consequences of an incorrect reading may be disastrous. In addition to the responsibilities of the manufacturer, the user also has responsibilities for routine care and maintenance, and especially for calibration. Manufacturers and their associations have provided guidance to assist users. More sensitive sensors are available based on galvanic and amperometric cells, surface acoustic wave semiconductor sensors, etc. These are in common use for reactive and highly dangerous gases including hydrogen sulfide, chlorine, etc. They can be programmed for read-outs of average and peak concentrations and often include an alarm.

Stand-alone gas-chromatographic detectors are used to indicate classes of organic vapors without discrimination, and the addition of a portable gas-chromatograph improves selectivity. Miniaturization of these devices is underway, and will make them more applicable to routine monitoring. Infrared spectrophotometers can also give information about the types and concentrations of chemicals present in the environment. While all these instruments have problems of sensitivity (typically not better than $1 \mathrm{ppm}$ ) and selectivity, their biggest drawbacks for routine use are size and weight, and, most importantly, cost. All of these issues are the subject of continued research and improvement. Instruments with the ability to provide instantaneous output and with data-logging and averaging capabilities have enormous potential for identifying high exposure situations in real-time so that administrative and engineering controls can be applied. Therefore these instruments have advantages over techniques that only provide an integrated result at some point after the exposure has occurred, provided they can be produced to meet other criteria of sensitivity, specificity, accuracy, user acceptability, and cost.

\section{Detector tubes}

A very useful item in the arsenal of occupational hygiene assessment techniques is the detector tube. When one of these tubes is exposed to the air a color is developed along the length of the tube that is proportional to the concentration of the contaminating species. The manufacturers painstakingly research the color reactions to be specific and sensitive for a particular chemical species, but cross-reactions are common, so that many tubes respond to classes of chemicals rather than one chemical alone. Not all reactions are sensitive enough for comparing results to exposure limit values, but instead they may be used to warn against acutely hazardous atmospheres. Air is pulled through the tubes using either a piston or bellows pump (caution: do not attempt to exchange pump styles) for an immediate result, although some pumped and diffusive tubes for long-term monitoring are available. The chemicals often have a finite shelf-life, and sometimes specific requirements for storage. Detector tubes are moderately expensive, and the tubes providing immediate results are not commonly used for comparison with long-term exposure limits, as the many tests that would be required for proper coverage of the exposure period would be too costly. Nevertheless, these tubes are very useful for workplace surveys, particularly in complex situations where multiple costly instruments would be required.

\section{Whole air samplers}

The simplest way to take a sample of an atmosphere is to enclose an aliquot in an inert container. Glass containers have given way to polymer bags and stainless steel canisters. Various polymers have been used for bags including Teflon ${ }^{\text {TM }}$ (polytetrafluoroethylene), Tedlar ${ }^{\mathrm{TM}}$ (polyvinyldifluoride), and Saran $^{\mathrm{TM}}$ (polyvinylidenechloride), and Mylar ${ }^{\mathrm{TM}}$ (polyethylene terephthalate) layered with aluminium foil. Polymer bags are used in some methods where analysis can be performed on-site, or where a polymer bag is a suitable container, such as with exhaled breath monitoring. However, the biggest drawback of using a bag is loss of contaminant, which may reach significant levels for some compounds within 24 hours of sample collection. There are other issues of bag collection, including the limitations of the bulk of the bag, re-use and the maintenance of bag integrity, potential contamination from the polymer and fittings, means of filling the bag (passing air through a pump can involve sample losses or potential contamination), photochemical reactions, and pressure differences between the site of collection and analysis. Stainless steel canisters, passivated or lined with an inert material such as fused silica offer an alternative to bags. These rigid containers can be thoroughly cleaned and evacuated before use, and are filled either instantaneously, or over a designated time period using a critical orifice. These containers were originally designed to take 6-liter samples of outdoor air, but personal 0.5-liter canisters are now also available. Many chemicals have been shown to be stable in such containers for long periods of time. Analysis is by overpressuring the container with an inert gas in the laboratory and either injection into a gas chromatograph directly through a gas sampling valve, or after concentration in a cold trap followed by thermal desorption.

\section{Bubblers (Liquid sorbents)}

After the impinger (see below) was developed for particle sampling, it was realized that the injection nozzle could be replaced by a glass frit which would produce a large bubble population. Gases and vapors diffuse quickly across a small bubble, and then across the bubble interface to dissolve in the surrounding liquid. The efficiency of absorption in such a device varies with the amount collected in accordance with Henry's Law of solubility, but the situation becomes much improved when a reagent is added to the liquid to react with the absorbed gas or vapor, such that the concentration in solution is maintained essentially close to zero. Unfortunately, even though these devices have been miniaturized, having something bubbling away under one's chin is still not popular with workers, and problems of handling liquids, including the possibility of breakage and spillage, make bubblers unpopular with field hygienists. Therefore the use of impingers has been declining over the years, although they still are employed when a solid sorbent is not available. Alternatives to bubblers have included liquid sorbent diffusion badges, where the liquid is held in place by a barrier through which gases and vapors can diffuse, although these are rarely seen today. Examples of gases and vapors that have been sampled this way include chlorine, methanol, and aliphatic amines.

\section{Solid sorbent samplers}

Pulling air through tubes containing a solid sorbent material for later analysis has a long history. The sorbent bed is contained in a tube, called a sorbent tube, which is a few 
centimeters long and a few millimeters wide. In this technique, air containing gas and vapor molecules passes through the bed and the molecules are removed by adsorption or chemical reaction. Small battery-powered air pumps are attached to the belts of workers and the inlets of the pumps are connected by flexible tubing to the sorbent tube, which is attached to the lapel of the workers shirt, or some equivalent point near to the mouth or nose. The pumps pull air at a fixed, calibrated flowrate through the sorbent tube. At the end of the designated sampling period the pump is switched off and the start and stop time and flow-rate are recorded. Typically the flow-rate has been calibrated at least at the beginning and end of the sampling period, if not more often, and so the total volume of air sampled can be recorded. The sorbent tube is then capped and sent to the laboratory for analysis, after which the total mass of each volatile organic compound (VOC) is divided by the air volume to give the concentration in mass per unit volume from which the volume ratio concentration can also be calculated.

Adsorption is a surface phenomenon and the amount of surface available for adsorption is critical. Not all the sorbent surface is available for adsorption, particularly when the surface of the sorbent is porous, as molecules might be too large to enter the pores (this is known as the "molecular sieve" effect). Since the amount of surface is related to the porosity of a material, sorbents with very high surface areas tend to have pores on the order of molecular dimensions. Although, initially, silica gel was used for aromatic hydrocarbons, the presence of competitive water vapor molecules in air made the collection and analysis of volatile, polar compounds difficult. In the early days of chemical exposure regulation, there was a need to develop quickly a comprehensive technique for sampling and analysis of the majority of volatile organic compounds in air, and the combination of charcoal adsorption, desorption with carbon disulfide, and analysis by gas-chromatography and flame ionization detection was very attractive as it maximized the number of volatile organic chemicals that could be measured with a single, simple procedure. Charcoal has a combination of pores of different dimensions, and so adsorbs a wide variety of volatile organic compounds, and carbon disulfide elutes early in standard gas-chromatographic methods and has a low background on the flame ionization detector, which is sensitive to nearly all organic compounds over a wide linear range of concentration. This is still the commonest technique in use for sampling and analysis of volatile organic compounds today.

Many other sorbents are available. Charcoals from various sources have different applications. Silica gel is used for some highly polar organic compounds and inorganic acid gases. Zeolite and carbon molecular sieves have applications in sampling very volatile compounds, including some that are gases under normal conditions (e.g. nitrous oxide). Porous polymers come in different pore sizes and sorbent capacity, and in a range of polarities, and can be tailored for specific classes of chemicals. Tube size and, therefore, sorbent quantity, can also be varied. Traps for water vapor, ozone, etc. can also be added.

Active and passive sampling. The traditional practice of sampling as explained above is by pulling air through the sorbing medium using a pump. Pumps have disadvantages of cost, bulk and weight, noise, potential for break-down, and need for calibration. Pump manufacturers have responded to these issues over the years by significantly reducing bulk and weight, and by making the pumps quieter and more reliable, and many pumps now have precise and accurate internal flowmeasurement devices, and yet, in real terms, they are hardly more expensive than 20 years ago. Nevertheless, so-called "passive" or diffusive devices, which operate by diffusion of contaminant molecules through a defined path to the sorbing medium, have advantages of user acceptability and ease of use (which makes them able to be used without much training, even by workers themselves). These devices are no longer new to the market, having been in existence for about 25 years, but their acceptance has been delayed by a lack of government validation and endorsement. Nevertheless, the accumulation of validation studies and experience has caused professionals to become more comfortable with their potential applications. Diffusive samplers are inherently more expensive than the pumped counterparts, so that they are most useful when capital outlays for pumps and calibrators is not possible or where only a few samples are required. In addition, when diffusive samplers can be used without the employment costs associated with calibration, the cost-benefit equation can swing in their favor.

Diffusive samplers are available from many companies. They cannot be used for some extremely volatile or many semivolatile compounds, or where aerosol must also be characterized. Many validation studies have provided experimentally validated uptake rates, which have been collected and published (e.g. the U.K. Health and Safety Executive methods for the Determination of Hazardous Substances method 88). These studies have also verified that calculations used to provide uptakes rates in the absence of experimental data are frequently within $5 \%$ of the experimentally verified figure. Their biggest drawback is that the uptake-rate is fixed by design, and thus they may not be useful at the extremes of a concentration range, either lacking sensitivity, or becoming overloaded, while the flow-rates of pumps can be varied over at least an order of magnitude to cover a wider range.

Solvent and thermal desorption. Collecting gases and vapors is only the first step in analysis. The second step is to recover the collected species in a form that is presentable to an analyzer. This is known as sample recovery, or desorption when the compounds were adsorbed in the collection step. Most sorbents are desorbed using solvent displacement, although the choice of solvent is very important, as it must be compatible with further steps of the process, especially considering the solvent molecules will outnumber the collected molecules by about $1000: 1$. In the case of charcoal, carbon disulfide has a unique combination of good penetration into pores, high heat of adsorption on charcoal, and relatively good solvation ability, at least for non-polar vapors. These properties, combined with the aforementioned fast chromatographic elution and low background on the flame ionization detector, have made it a very popular desorbing solvent. Polar modifiers such as isopropanol or butanol may be added to improve the recovery of polar compounds, provided they do not interfere with the analysis. Alternative solvents are necessary where carbon disulfide, even with modifiers, is a poor solvent, or where it reacts with or masks chemicals of interest, or where it introduces contamination. Methanol with methylene chloride, and dimethylformamide have been used in place of carbon disulfide.

Thermal desorption is a technique that avoids the use of solvents in the recovery process. Molecules are driven off from the sorbent by the application of heat. Re-concentration normally is necessary, but the entire process may be automated and integrated directly with a gas chromatograph. Thermal desorption is appropriate for almost all volatile organic compounds that can be chromatographed, provided the sorbent can be matched with the requirements of collection and recovery. Water vapor molecules are also trapped and released. If present in large quantity they can interfere with the chromatography and detection of other molecules, so that water management systems, for example a dry purge facility, may be useful.

Charcoal is not an effective sorbent for thermal desorption, as compounds are held too tightly in the micropores, and thermal breakdown, catalyzed by inorganic constituents within the charcoal, tends to occur before release. Polymer sorbents 
such as Tenax ${ }^{\mathrm{TM}}$ (poly-(phenyl-p-phenylene) oxide) and Chromosorb $^{\mathrm{TM}} 106$ (poly-styrene/divinylbenzene) have been used, as well as graphitized carbons and carbon molecular sieves (for the most volatile compounds). The criteria for efficient collection and the criteria for efficient release are opposed, so that a sorbent must be matched within a range of contaminant volatility and concentration. Multiple sorbent beds, where the sorbents are selected to cover different volatility ranges, allow a greater range of contaminants to be collected. The advantages of thermal desorption include enhanced sensitivity, as no solvent dilution is involved. Conversely, it may be easy to overload the sampler or the detector without adequate precautions. Splitting and re-collecting the desorbed sample, prior to entry into the gas chromatograph, may help to avoid this problem. Thermal desorption tubes are expensive and re-usable but must be handled carefully to avoid contamination. The tubes themselves may be re-used indefinitely if in good repair, but sorbents will need to be removed and replaced at some point. The oven of a thermal desorber usually is tailored to a specific tube dimensions. The user should contact the analytical laboratory before sampling to ensure the appropriate size tubes are available.

Chemical reaction. Some chemicals are too reactive to be sampled on charcoal. Some may need to be transformed by derivatization into substances that are more easily separated or detected in the analytical stage. In these cases, it is common to sample such chemicals by reaction. The reactant can be applied to the surface of the fibers that make up a glass or quartz-fiber filter, or to the surface of a sorbent in a sorbent-tube (as in the case of detector tubes, above), or added to the liquid in a bubbler or impinger. Fast and efficient reactions are preferred, but air flow-rate through the medium may have to be reduced to ensure adequate contact time. Wetting agents may also need to be applied with the reactant. Some important examples include sampling ethylene oxide (conversion to bromoethanol with hydrogen bromide), aldehydes (reaction with 2,4dinitrophenylhydrazine or hydroxymethyl piperidine), isocyanates and amines (many different derivatizing agents in use). Just as with detector tubes, chemically-coated sorbent tubes may have a finite shelf-life or specific storage requirements.

\section{Methods of assessing exposures to aerosols}

Assessing exposures to aerosols presents different challenges from sampling gases and vapors. There is much debate, even today, about the appropriate metric. The relative importance of mass, number, size and surface or bulk composition, and even the viability of microorganisms may differ for specific situations.

\section{Direct-reading instruments}

The comments regarding the advantages and disadvantages of direct-reading instruments for gases and vapors generally also hold true for the similar devices that have been developed for measuring aerosols. The most popular method of detection for direct-reading instruments for aerosols has been reflectance. Light-scattering devices are cheap, although their response varies with the light-scattering properties of the aerosol under examination, so that they are only rarely used for assessing environments against a standard, due to the difficulty of calibration. Their response also varies with particle size. Recently, piezo-balance detectors have become popular. Since they react to mass, their read-out can be compared directly to standards given in mass terms. However, part of the collected mass may be water vapor, and so the devices normally are maintained above ambient temperature, which can lead to losses of volatile aerosol. Condensation nuclei counters are very popular for very fine (including "ultrafine") aerosols. Other technologies are being developed, and the pace of this development has received an additional impetus from the need to provide equipment for responding to biological threat agents.

\section{Impingers}

The midget impinger was designed as a method for collecting aerosol in mines, at a time when particle numbers were considered important and occupational exposure standards were couched in number terms (e.g. millions of particles per cubic foot). Particles enter a glass tube, and are accelerated into a liquid, only to collide with an impaction plate (which, today, is the base of the impinger glassware). The particles lose momentum and become wetted, generally remaining in the liquid. To count the particles, a known aliquot of liquid is pipetted onto a microscope slide, and the particles are counted under a graticule. The collection efficiency of impingers for large particles has not been well-researched, but is likely to fall with increasing particle-size. At the other end of the spectrum, very small particles can be lost first by becoming attached to bubbles, followed by release into the exit air stream when the bubbles burst. Impingers are rarely used for particle collection today, except in the special case of biological aerosols (fungal spores, bacterial cells, etc.) whose sizes fall generally in the region of efficient particle collection, and where viability of the organisms is enhanced by collection in a liquid medium. A special area of application is where it is desired to stabilize chemical moieties on particles through chemical reaction (e.g. isocyanates). Wetting of particles in an impinger solution allows better contact of the reactants than when the reacting chemical is coated onto a filter medium. However, a coated filter is often placed in-line behind the impinger to account for the aforementioned small particle losses.

\section{Filters and cassettes}

It is much more common today to calculate airborne concentrations of particles in terms of mass per unit volume. Particles collected from a known volume of air may be weighed directly, or analyzed chemically with the result given in mass terms. Collection of particles on a filter is the preferred method. Several types of filters are in common use, including glass and quartz fiber, and various polymeric membrane filters. Several considerations can affect the selection of the most appropriate filter for a specific situation. Such considerations include flowrate and pressure drop across the filter, stability and blank levels on the filter, and the method of recovery and analysis. As the rate of air-flow through a filter increases, so does the pressure drop across the filter. For vacuum pumps this is rarely a problem, but even personal samplers rated to a particular maximum flow-rate may not be able to maintain that flow-rate for long periods of time against a significant pressure drop without premature battery discharge. For fiber filters, pressure drop is rarely an issue, but for membrane filters, the smaller the membrane openings (pore-size), the higher the pressure drop. This problem is exacerbated by the size of the filter; for example, a $25 \mathrm{~mm}$ filter has one-quarter the area of a $37-\mathrm{mm}$ filter, and so the linear velocity for a specific flow-rate is higher, and, therefore, so is the pressure drop across the filter. Overloading of the filter with excessive dust deposits can add to the pressure drop problem. Filters are held in a sampling head, sometimes known as a cassette. The sampling head may be open to the environment directly, but, more usually, the sampling end is modified with some form of size-selective inlet, to ensure collection of particulate within a specific size-range. There is a wide range of cassettes and size-selective inlets available, and different products may be specified in individual standards and regulations. The standard sampling head in the USA is a poly-styrene/acrylonitrile cassette, which houses a 37-mm diameter filter. The outlet end is connected to the inlet of a sampling pump. The inlet cap of the cassette has a 4-mm 
diameter entry orifice. This is known as the "closed-face" configuration. Removing the inlet cap and replacing it with a retaining ring is known as the "open-face" configuration. The cassette normally is held in place near the lapel of the worker's shirt, or equivalent location, pointing downwards at approximately a $45^{\circ}$ angle. This cassette may be available in transparent, colored, opaque, and in other polymeric materials, including acid-proof and static-dissipative ("conductive") materials. Special internal cassettes have also been designed to hold the filter within the standard cassette housing. These internal cassettes are weighed in their entirety, to ensure capture of particles that otherwise might be lost on the wall of the standard cassette, and they may also have tamper-proof features.

The capture efficiency of a filter is related to its pore-size. For membrane filters with etched holes, the filter acts partially as a sieve, and particles smaller than the rated pore-size can pass through the filter. This is true of mixed cellulose nitrate/acetate ester (MCE or MEC) and polycarbonate filters. For these filters there is a trade-off between pressure-drop and particle loss. Pore-sizes of $1 \mu \mathrm{m}$ or slightly less are the most effective filters which can be used at high flow-rates with personal sampling pumps, but some sub-micron particles may still be lost. On the other hand, many different collection mechanisms operate in the depth of a fibrous filter to allow efficient collection of even fine particles. True fibrous depth filters used in air sampling, such as those made from glass and quartz fiber, are not rated in terms of pore-size at all. Polyvinylchloride (PVC) and Teflon ${ }^{\mathrm{TM}}$ filters share characteristics of both membrane and fibrous filters. Although they are rated in terms of pore-size, the size is determined based on filtration of solid suspensions in liquid. Particles in liquid suspension are not subject to exactly the same filtration mechanisms as those in air, and PVC filters rated as high as $5 \mu \mathrm{m}$ are effective in removing the majority of particles smaller than $5 \mu \mathrm{m}$, with lower pressure drop than other membrane filters.

The calibration of filter cassettes in general requires there be no leakage other than flow through the inlet of the cassette and through the filter. Infiltration of air through the body of the cassette can, and should, be checked using a commerciallyavailable leak tester, in order to avoid bias. Leakage in batches of cassettes from different manufacturers has been noted. The gel-bands that are often used around cassettes provide useful surfaces for writing but do not provide adequate leak-proof seals.

The stability and blank level requirement depends heavily on the type of analysis. The most common analysis is gravimetric. Therefore any environmental factors that can affect the weight of filters are important. The most important factor affecting weight stability is humidity. Water vapor is present in the air, usually in tens of thousands of parts per million, and these levels can fluctuate considerably over time. Filters that absorb water vapor may change weight significantly in a relatively short time-period, or when equilibrating to a new set of conditions. Glass fiber and MCE filters are prone to absorbing water vapor, and it is usually considered appropriate to desiccate these filters, or at least to equilibrate them to a standard humidity, prior to weighing. Desiccation can cause part of the collected aerosol to evaporate, and this is difficult to determine in practice. In addition, dry MCE filters are prone to static effects, which can make them difficult to handle and weigh accurately. Therefore, equilibration often is preferred to desiccation. PVC filters absorb little moisture, and do not need so much equilibration, and are much preferred for gravimetric analysis. However, it is necessary to be careful of mixed polymer PVC filters (e.g. acrylic co-polymers) if the co-polymer is polar. A recent study by the UK Health and Safety Laboratory ("Round Robin Filter Weighing Exercise" J. McLister, P.R. Stacey and G. Revell, May 2001, HSL, Broad Lane, Sheffield, UK) compared the precision of weighing different loadings on PVC and glass fiber filters. They found, not unexpectedly, that the precision of weighing began to become unacceptable at loadings below $1 \mathrm{mg}$, but found the major decrease in precision for PVC filter weighing occurred at lower loadings $(<0.2 \mathrm{mg})$ than that for loaded glass fiber filters $(<0.5 \mathrm{mg})$. Incidentally, less variation and increased accuracy was found with a six-decimal place over a five-decimal place balance.

Both PVC and MCE filters can absorb some volatile organic compounds from the atmosphere, so for highly sensitive analyses of ambient air samples, Teflon ${ }^{\mathrm{TM}}$ filters are used. These filters are very expensive and require careful handling because of static effects. The cost, coupled with the high pressure-drop across the filters, makes them less often used in industrial hygiene analyses, but they are used in certain methods where it is important not to include volatile organic compounds, such as in the analysis of metalworking fluids. Note that Teflon ${ }^{\mathrm{TM}}$ filters with polymeric backing other than Teflon ${ }^{\mathrm{TM}}$ may still absorb volatile organic compounds.

Some types of cassettes used to house the filter and to ensure all dust collected by the sampler is included in the analysis are also affected by ambient humidity. Since these cassettes are weighed in their entirety with the filter, any change in mass will seriously affect detection of changes in the filter mass. Internal cassettes that are used in the $37-\mathrm{mm}$ closed-face cassette are made of plastic or aluminium foil that is relatively stable and light-weight. The plastic cassette that is used in the Institute of Occupational Medicine or IOM sampler (a sampler designed to meet the inhalable sampling convention, see below, designed at the Institute of Occupational Medicine, Scotland) is made of a conductive plastic that is highly water absorptive, and that can take many days, or even weeks, to equilibrate in a desiccator. The problem this causes for accurate gravimetric analysis is now well-known, and it has been suggested that field blanks can be used to correct for this variability, but corrections that are so large as to be similar to or larger than the corrected mass are undesirable, and multiple blanks are required for most accurate correction. In addition, it appears that different batches of cassettes from the same manufacturer have different responses, so that the blanks must be identified as coming from the same batch as the field samplers. The HSL study mentioned above concluded that unless the potential loadings were very large then any gravimetric data produced by weighing filters in plastic IOM heads is likely to be of dubious value. Stainless steel sampling heads are not affected by humidity but cannot be tared on many six-place balances as they are too heavy.

As mentioned above, most aerosol samples are analyzed gravimetrically, but gravimetry is a very crude technique, which does not differentiate between a chemical of specific concern and other sources of aerosol in the environment, nor does it differentiate between chemical species, and neither does it have sufficient sensitivity to be used for chemicals with exposure limits in the microgram per cubic meter range, as is the case with many metal salts. Chemical analysis is much more specific and sensitive. Glass fiber filters can absorb metals, so cellulose ester membrane filters generally are used for metals analysis. These filters can be completely digested in hot, concentrated acid. However, in addition to the safety issues surrounding the use of hot, concentrated acid, the digestion process is also timeconsuming. Digestion of some metals is also possible in much shorter times using dilute acid at room temperature with sonication. In this case the filter is not digested, but the particles are lifted off the filter and dissolved by the extremely high energy of ultrasound. Where good recovery efficiency can be demonstrated using sonic extraction with PVC filters, then it is possible to also obtain a result for total dust as well as for the specific chemical elements. PVC filters may dissolve partially in this process, but this not likely to be a problem with graphite furnace atomic absorption spectrophotometry. Microwave digestion has also been used with MCE filters. 
For soluble salts, an International Standards Organization (ISO) Standard 15202-2 has been published on extraction procedures (see: K. Ashley, "International Standard Procedure for the Extraction of Metal Compounds Having Soluble Threshold Limit Values", Appl. Occup. Environ. Hyg, 2001, 16, 850-853). Two methods have been favored in the past, extraction in pure water (a "chemist's strict definition" of solubility) and extraction in diluted hydrochloric acid (an attempt to define "bioavailability"). Deionized water is the commonest solvent used in U.S. standard methods. The ISO Standard has formally adopted this procedure, while allowing individual countries to specify alternative extraction media, including dilute hydrochloric acid. The ISO procedure assumes sample collection on filters and mentions potential issues of incompatibility. For example, hexavalent chromium can be reduced to trivalent chromium on some filters (PVC appears to be better than MCE) and silver can react with chlorides from PVC filters (Teflon ${ }^{\mathrm{TM}}$ is better).

Some filters are used for surface analysis by microscopy. MCE filters are used for fiber analysis by phase-contrast microscopy, while polycarbonate filters are popular for microorganisms (black filters are useful for their contrasting background for epifluorescence microscopy). Polycarbonate filters are often used for endotoxin analysis. Some are certified to be free of contamination. Silver filters are used in some US methods for the analysis of silica, as the silver X-ray diffraction peak can be used for calibration.

Glass fiber filters are often used as supports for chemicals, which react with airborne vapors and particles. Sampling chemicals in this manner is very useful when either the chemical is too reactive to be collected by adsorption on charcoal tubes, or when the resulting reaction product (called the derivative) is either more easily separated or quantified. Some methods make use of all these advantages. Compounds suitable for this type of sampling include isocyanates, aldehydes, and organic amines. The fiber filters can be loaded with large quantities of the reactive chemical and provide extra depth for increased contact time.

Quartz fiber filters are a purer form of glass fiber filters that can be cleaned by heating to very high temperatures. This allows their use in methods for diesel exhaust that measure carbon collected on the filter. Heating the filter burns off organic matter that would interfere with the analysis.

Careful selection of filters is a very important part of a hygiene survey. Filters are not all the same, and even filters that are nominally similar may differ in important respects (e.g. pure homopolymer PVC and mixed-polymer PVC, Teflon ${ }^{\text {TM }}$ with and without backings, etc.). The analytical laboratory can normally provide advice as to which is the most appropriate for a particular purpose.

\section{Size-selective sampling}

Size-selective sampling is based on the simple premise that particles can deposit in different parts of the human airways system, and may cause different health effects depending on where they settle. Some examples include nasal perforation from hexavalent chromium salts, upper airways irritation from acid gases, and alveolar silicosis from fine silica particles. Both particle penetration and deposition depend on the aerodynamics of the particle in the airway, and current criteria for size-selection are based on penetration, rather than deposition. For large particles this is appropriate, since the inlet efficiency of the human mouth and nose are significant factors, but for very small particles, there is a deposition minimum around $0.3 \mu \mathrm{m}$ that is not taken into account. This deposition minimum is a candidate for defining the upper limit of a fraction known as "ultra-fine", although other size limits have been proposed for this class of particle. Very fine particles of molecular dimensions tend to behave more like gases and vapors, so that deposition in the head airways by diffusion may be a significant capture mechanism. Size-selective sampling has been adopted by many organizations, and reference may be made to the Comité de Européen Normalisation (CEN) standard EN 481, or to the International Organization for Standardization (ISO) standard 7708.

Respirable. The fact that fine particles will penetrate to the deepest recesses of the lung, and there cause several forms of pneumoconiosis ("dusty lung") has been known for a long time. Many occupational exposure standards exist for particles in the $<10 \mu \mathrm{m}$ size-range. However, the actual point of demarcation is still a subject of dispute. Whether one follows the British Medical Research Council or BMRC convention $(50 \%$ penetration efficiency at $5 \mu \mathrm{m})$, the old American Conference of Governmental Industrial Hygienists or ACGIH convention $(50 \%$ penetration efficiency at $3.5 \mu \mathrm{m})$, or the compromise ISO convention ( $50 \%$ penetration efficiency at $4 \mu \mathrm{m}$ ), it is likely that the size-separation device of choice will be a cyclone. Cyclones have a penetration efficiency curve similar to respirable efficiency curves over the range $1-10 \mu \mathrm{m}$. Adjustments to the particle penetration efficiency can be made by altering the flow-rate through the cyclone. However, it is necessary to be sure the correct flow-rate is applied to each particular cyclone type in order to provide the specific sizeselection requirement. Cyclones (and other aerosol samplers) may be compared by reference to a size-selective convention by calculating the total mass bias that would occur for an aerosol of unimodal log-normal distribution with a specific mass median aerodynamic equivalent diameter (MMAED) and geometric standard deviation (GSD). A "bias map" can be drawn, connecting points of equal bias, for a space covering a realistic range of MMAED's and GSD's. Silica and coal dust are examples of materials with respirable exposure standards.

Thoracic. The thoracic convention was defined much more recently than the respirable convention. It relates to particles and droplets that can pass through the head airways, and have a significant probability of deposition anywhere in the lung, including the upper thoracic airways. There are few exposure standards relating to the thoracic convention, but candidates include those chemicals producing upper airways irritation (e.g. acid mists) and those chemicals involved in reactive airways disease or hypersensitivity pneumonitis and asthma. The NIOSH has put forward a thoracic Recommended Exposure Limit (REL) for metalworking fluids. A cyclone that operates in accordance with the thoracic convention has been developed, although the flow-rate is rather low. Even though the penetration curve for the thoracic convention is not as sharp a cut as that of an impactor, $10 \mu \mathrm{m}$ single-stage impactors can be used for most commonly encountered aerosol distributions without excessive bias. The 37-mm closed-face cassette (see below) has also been evaluated for use in sampling metalworking fluids according to the thoracic convention. A correction factor has been proposed to adjust the result, but there is some discussion as to whether a single correction factor is applicable to all industrial situations.

Fibers are a unique class of aerosols that require specific methodologies for collection and analysis. Cotton dust sampling, for example, requires a special sampler, known as a vertical elutriator, and asbestos sampling uses a particular cassette conformation with open-face and extended cowl. In general, it is thought that the thoracic convention may be applicable to these materials.

Inhalable. For many years, exposure assessments to most dusts have focused on collecting all airborne particles ("total" dust). However, the upper size limitations of most samplers were not considered until recently, because of an assumption that particles above a certain size would not remain airborne 
long enough to be inhaled, due to their tendency to settle under the influence of gravity. For particles up to about $30 \mu \mathrm{m}$ in MMAED, most samplers are relatively efficient collectors. However, the proposed "inhalable" convention, to include all particles with potential for penetration into the human airways system, including those particles that would likely deposit in the head airways, extends up to $100 \mu \mathrm{m}$ MMAED. In the range between 30 and $100 \mu \mathrm{m}$ MMAED, most samplers are not as efficient collectors as the convention demands, and they therefore under-sample when coarse particles are present. Research to compare existing "total" aerosol samplers with "inhalable" aerosol samplers, and to develop new "inhalable" samplers, continues. The IOM sampler mentioned above is the only sampler developed a priori to meet the inhalable convention, although some others, including one from Germany ("GSP") and another from France ("CIP-10"), may meet the requirements of the convention.

For exposure situations where coarse particles are common the impact of switching to sampling in accordance with the inhalable convention may be profound. The majority of samplers specified under the national guidance or regulations in most countries do not collect coarse particles as efficiently as the convention demands. Sampling with a sampler that meets the inhalable convention, without change in the numeric value of the airborne material concentration standard, may cause the standard to be exceeded more frequently. The question then arises as to whether the existing procedure is less protective. Greater numbers of large particles are usually associated with increased numbers of smaller particles, and this relationship means small particles may be a predictor of large particles exposures. Thus, if exposure standards were set with reference to dose-response studies using the existing sampler, and the results from the existing sampler can be multiplied by a standard correction factor to be equivalent to the results from an inhalable sampler, then the exposure standard itself should be similarly adjusted upwards. However, it is not clear that this relationship is always true, and there are other contributing factors. For example, samplers that operate in accordance with the inhalable convention might also incorporate particles greater than the upper limit of the inhalable convention. The presence of such "ultra-large" particles can seriously bias the measurement

Sometimes, it is useful to obtain more information about the particle size distribution in a workplace than is provided by the size-selective conventions described above. Instruments such as the Aerodynamic Particle Sizer (APS) can be used, with appropriate calibration, to provide particle size-distribution information over a wide range of particle sizes. However, this instrument is not a personal monitor, is quite expensive, and does not measure mass directly. A stack of impactor plates ("cascade impactor") can be worn on the body, and gives mass concentrations, although in larger size-range increments. Such devices can detect the presence, for example, of bi-modal aerosol distributions, which might escape discrimination by other methods. More recently, devices which incorporate the three health-based size-selective fractions have become available, such as the IOM or GSP sampler with foam inserts, or the "Respicon"TM virtual impactor.

\section{Mixed exposures}

The term "mixed exposures" has been applied to exposures to mixtures of different vapors, e.g. multiple solvents. If the components of such a mixture affect different target organs, then each should be assessed against its own standard, but if there is interaction between the components, it is necessary to determine whether the interaction is simply additive, or synergistic, before determining the degree of hazard associated with the exposure. The term has also been applied to exposures to chemicals and physical agents, and, for example, it is known that certain solvents have an effect on the severity of damage from noise. Another common usage for the term mixed exposures is exposure to chemicals that exist simultaneously in the aerosol and vapor phases at the ambient conditions. Many of these compounds fall into the class of "semi-volatile" compounds, e.g. polyaromatic hydrocarbons, and organophosphorous pesticides, but, generally, chemical compounds are present in both aerosol and vapor phases because of their mode of use or occurrence in industrial processes, for example, isocyanates and pesticides applied through spray, or metals and polyaromatic hydrocarbons condensing from a hot vapor phase.

Generally, it is assumed that the mass present in both forms will contribute to the overall toxicity, so the focus is on sampling the total. However, control measures may vary depending on the physical state of the contaminant, so some indication of the relative proportions between the phases is often useful. Pre-filters are often used to collect particulate, with some form of sorbent back-up to collect the vapors. Since semi-volatile vapors are readily condensed, sorbents are often selected with large pores and relatively low surface areas (e.g. Amberlite XAD2 resin, or polyurethane foam). The main advantage of polyurethane foam is the very low pressure drop across the medium allowing high flow-rates and collection from large air-volumes for maximum analytical sensitivity. The simple filter/sorbent combination cannot be used to determine the exact nature of the partitioning between aerosol and vapor in the air, because evaporation from collected aerosol adds to the vapor fraction, and condensation of vapor on the filter or collected aerosol adds to the aerosol fraction. A diffusion denuder prior to the filter can be used to collect vapor upstream from the aerosol, although it increases the size of the sampling system. A specially designed impactor system has also been developed for separating aerosol and vapor.

\section{Dermal exposures}

In many exposure situations there is the potential for a contribution to the overall exposure from a "dermal" route. This may involve true dermal exposure by contact of the chemical with the skin, or other membranes, such as the eyes, and its subsequent penetration, or the skin may also be a mechanism for transporting chemicals to the mouth where they are subsequently ingested. The many factors that control dermal contact and penetration are currently under intensive study. For example, the role of clothes in holding substances against the skin for long periods of time is important. The methods for assessing dermal exposure involve investigating the extent of contamination of the environment, clothing and the skin, and may involve wipes of surfaces, collecting washes from hands, or placing adsorbent patches on the body. Filter cassettes have also been used as vacuum samplers for surfaces. These methods can involve chemical analytical techniques that produce a quantitative result (although the assumptions of sampling generally cause the overall conclusion to be semi-quantitative), but they can also involve colorimetric reactions that produce a qualitative warning. Fluorescent imaging of chemicals, either by auto-fluorescence or adding a fluorescent tracer, provides dramatic evidence of contamination, which also can be treated semi-quantitatively, according to the depth of the fluorescence.

\section{Biomonitoring}

The methods mentioned above provide surrogate measures of an individual's exposure. Biological monitoring has the advantage of measuring responses which depend only on the individual's actual exposure. Biological indicators that have been used include the presence of the chemical itself, or a metabolite, in blood, urine, or breath, and measures of the physiological effects of exposure, such as the depression of acetylcholinesterase following exposure to organophosphorous 
pesticides. Biomonitoring may also capture the results of exposure during periods when other forms of exposure monitoring have not been in use. Individual pharmacokinetics plays an important role determining the response to an applied dose, and special attention must be paid to ensuring biological specimens are collected in a consistent manner. For example, a particular sample may need to be collected during a work-shift, at the end of a work-shift, or even at the end of a week or month. Urine sample results may need to be corrected for urinary volume, and so on. Some biological indicators are guidelines only, but some are used as the basis for legislative control, such as is the case with biological lead and cadmium exposure indicators in the USA. Biological monitoring may be an alternative to other forms of exposure monitoring where it can be shown to be more cost-effective, or more practicable. However, frequent blood sampling, and even urine collection, is considered invasive by most workers. Exhaled breath monitoring may be a more acceptable procedure, and has been used for monitoring exposures to some chemicals, but more research is required before this becomes a universally adopted technique. When using biological monitoring to reconstruct exposure, it must be kept in mind that the results are confounded by the processing of the measurand through the body, so that they are more relevant to dosimetric considerations than exposure only.

\section{Validation}

Methods should be validated before use to ensure they give results with accuracy appropriate to the measurement task. Guidelines and regulations for the accuracy limitations of methods for different purposes are available. In theory, the sampling and analytical components can be validated separately. Typically, validation tests in the laboratory involve both components, by sampling from standard atmospheres. Since testing is highly variable, even within a single testing organization, users of a method should always review the reports of validation testing carefully. Validation should encompass the extremes of environmental conditions (including concentration of the hazard material) that the user would encounter in the field, although sometimes extrapolation may be warranted. Validation can assist in the determination of the accuracy (uncertainty) of a method, especially if multiple laboratories were involved, and multiple field sites, if field studies were performed. It should be kept in mind, however, that outside of a laboratory, and often, even within the laboratory, there is no traceable standard against which the method can be compared, only a previously validated method, with its own associated uncertainty. Validation protocols have been published and compared in an on-going effort to harmonize this activity. Following publication of the method, proficiency testing, available through many national and international organizations, can be used to ensure analytical competence. Some proficiency testing activities provide test materials prepared as simply as possible to exclude all but analytical variation. Others produce samples that are more life-like, including some where samples are taken from controlled atmospheres, which may even contain interfering substances purposefully introduced. Obviously the range of variation deemed acceptable will rise with increasing complexity of the sample. Other quality control measures that should be in common use, depending on the specific procedure, include analysis of blank reagents and media, analysis of field blanks, replicate sample analyses, analysis of certified reference materials, independent source calibration materials, multipoint calibrations, internal standards, and calibration verification standards. There should be quality specifications and the analysis of trends. Analysts should be trained and proven to be proficient. Laboratory accreditation schemes exist to make sure these goals are met.

\section{Bibliography}

Methods that may not currently be available on the internet

Health and Safety Executive, Methods for the Determination of Hazardous Substances, HSE Books, Sudbury, UK (series).

Deutsche Forschungsgemeinschaft, Analytische Methoden zur Prufung Gesundheitsschadlicher Arbeitsstoffe, DFG, Verlag Chemie, Weinheim, FRG, 1985.

Berufsgenossenschaftliches Institut fur Arbeitssicherheit: BIA Arbeitsmappe

Messung von Gefahrstoffen, Erich Schmidt Verlag, Bielefeld, FRG, 1989

Arbetslivsinstitutet, Principer och Metoder for Provtagning och Analys av Ämnen Upptagna på Listan över Hygieniska Grånsvården, Arbete och Hålsa, Vetenskaplig Skriftserie, 1997, 6, Solna, Sweden.

An excellent overview of monitoring techniques may be found in the articles, with references, in the section on Industrial Hygiene (ed. P.R. Michael, C.R. Glowacki and M. Harper) in the Encyclopedia of Analytical Chemistry: Applications, Theory and Instrumentation (ed. R.A. Myers), John Wiley, Chichester, UK, 2001, pp. 4577-4694

Articles:

Industrial Hygiene, Introduction

Aerosols and Particulates Analysis: Indoor Air

Carcinogens, Monitoring of Indoor Air

Chromatographic Techniques in Industrial Hygiene

Direct Reading Instruments for the Determination of Aerosols and Particulates

Metals in Blood and Urine: Biological Monitoring for Worker Exposure

Parent and Progeny Compounds in Exhaled Breath, Determination of

Sampling and Recovery Techniques for the Determination of Gases and Vapors in Air

Sensors in the Measurement of Toxic Gases in the Air

Spectroscopic Techniques in Industrial Hygiene

Surface and Dermal Monitoring

\section{Other useful texts}

American Conference of Governmental Industrial Hygienists, Air Sampling Instruments for Evaluation of Atmospheric Contaminants, 9th edn., ed. B. S. Cohen and C. S. McCammon, Jr., ACGIH, Cincinnati, OH, 2001.

American Conference of Governmental Industrial Hygienists: Bioaerosols, Assessment and Control, ed. J. Macher, ACGIH, Cincinnati, OH, 1999.

C. J. Maslansky and S. P. Maslansky, Air Monitoring Instrumentation: A Manual for Emergency, Investigatory, and Remedial Responders, John Wiley, New York, 1993.

J. R. Mulhausen and J. Damiano, A Strategy for Assessing and Managing Occupational Exposures, 2nd edn., AIHA Press, 1998.

S. A. Ness, Air Monitoring for Toxic Exposures: An Integrated Approach, John Wiley, New York, 1991.

S. A. Ness, Surface and Dermal Monitoring for Toxic Exposures, Van Nostrand Reinhold, New York, 1994.

Biological Monitoring: An Introduction, ed. S. S. Que Hee, Van Nostrand Reinhold, New York, 1993.

J. H. Vincent, Aerosol Science for Industrial Hygienists, Pergamon (Elsevier), Oxford, UK, 1995.

Aerosol Measurement: Principles, Techniques and Applications, ed. K. Willeke and P.A. Baron, Van Nostrand Reinhold, New York, 1993. 